\title{
Laser Spinning of Nanotubes: A path to fast-rotating microdevices
}

\author{
Petr Král ${ }^{1,2}$ and H. R. Sadeghpour ${ }^{2}$ \\ 1 Department of Chemical Physics, Weizmann Institute of Science, 76100 Rehovot, Israel \\ 2 ITAMP, Harvard-Smithsonian Center for Astrophysics, Cambridge, Massachusetts 02138
}

We show that circularly polarized light can spin nanotubes with $\mathrm{GHz}$ frequencies. In this method, angular moments of infrared photons are resonantly transferred to nanotube phonons and passed to the tube body by "umklapp" scattering. We investigate experimental realization of this ultrafast rotation in carbon nanotubes, levitating in an optical trap and undergoing mechanical vibrations, and discuss possible applications to rotating microdevices.

32.80.Lg, 62.25.+g, 78.67.Ch, 85.35.Kt

Carbon nanotubes [1] have unique mechanical and electronic properties with many potential applications [2]. They possess a huge Young modulus $Y>1 \mathrm{TPa}$, which adjusts their autonomous mechanical oscillations to $\mathrm{MHz}$ frequencies [3]. Moreover, their "molecular structures" remain naturally stable even at large deformations [ 4 .

Therefore, rotationally symmetric structures based on stiff nanotubes could form ideal piston-rods for nanoscale applications. In contrast to chemically driven bio-motors [5], spinning with $\mathrm{Hz}$ frequencies, such tubular structures could rotate very fast, if angular momentum is efficiently transferred to them and friction is reduced.

Small heteropolar molecules can be dissociated [6], if synchronously rotated with a dipolar laser trap, which accelerates its angular velocity. Larger molecules [7] and micro-particles [8] can be rotated by absorption of angular momentum from circularly polarized or "twisted" laser beams. Nanotubes are excellent candidates for this asynchronous driving, where the system rotational frequency is much smaller than the light frequency.

Here, we investigate ultrafast asynchronous rotation induced in nanotubes by excitation of their vibrational modes with circularly polarized light. The mode selection is restricted by radiational heating, since each photon absorbed by the tube transfers to it angular momentum $\hbar$ and energy $\hbar \omega$. The resulting heating can be limited in excitation of infrared (IR) $A_{2 u}$ or $E_{1 u}$ phonon modes, active in graphite [9] and nanotubes [10].

In Fig. 1 we show two schemes for spinning nanotubes. In the upper one, circularly polarized light beam propagates along the symmetry axis of the single-wall (SWNT) or multi-wall (MWNT) nanotube, levitating in an optical trap. The photon angular momentum is transferred to circularly polarized phonons, counter-propagating on the tube circumference (see Fig. 2), and latter passed by scattering to the tube body. The angular momentum of light could be also directly passed to the nanotube in excitation of its dense rotational levels. The resulting tube rotation with angular frequency $\omega_{\text {rot }}$ is mostly balanced

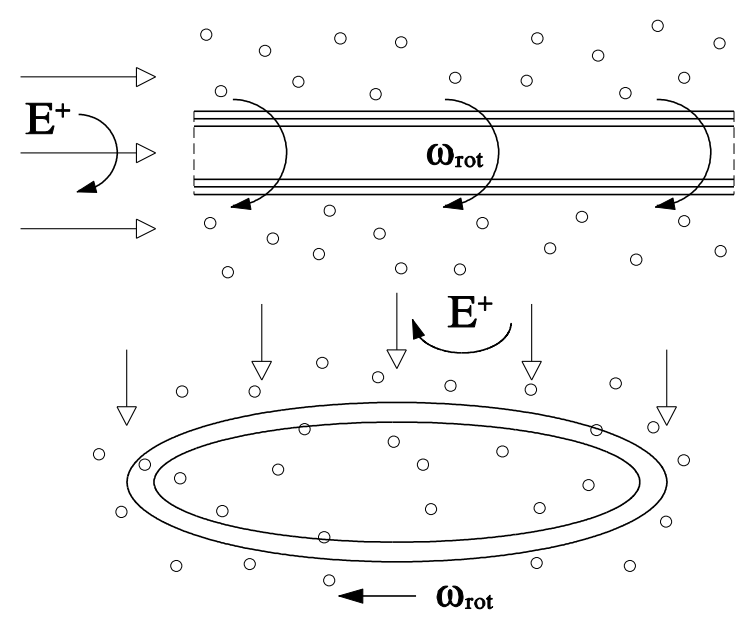

FIG. 1. Scheme for nanotube (up) and tubular ring (down) spinning with angular velocity $\omega_{\text {rot }}$ in a laser trap. Their rotation is induced by absorption of circularly polarized photons from a laser beam with intensity $\mathbf{E}^{+}$, propagating along the axis of rotational symmetry. Scattering of molecules with tubes damps the rotation.

by friction with the surrounding molecules. In the lower configuration, a closed nanotube ring [1] is analogously rotated by absorption of circularly polarized photons.

We can describe the excitation of nanotube phonons by circularly polarized light, and the subsequent relaxation, with the simplified Hamiltonian

$$
\begin{aligned}
H & =\sum_{\alpha} \hbar \omega_{\alpha} b_{\alpha}^{\dagger} b_{\alpha}+\sum_{\alpha_{ \pm}} \mu_{\alpha_{ \pm}} E^{ \pm}(t)\left(b_{\alpha_{ \pm}}^{\dagger}+b_{\alpha_{ \pm}}\right) \\
& +\sum_{\alpha_{ \pm}, \beta, \gamma}\left(c_{\alpha_{ \pm}, \beta, \gamma} b_{\alpha_{ \pm}}^{\dagger} b_{\beta} b_{\gamma}+H . c .\right)+H_{d} .
\end{aligned}
$$

The first two terms describe phonon modes $\alpha=($ band,$k)$ and coupling of the chosen IR circularly polarized optical phonons, with operators 12 $b_{\alpha_{ \pm}}^{\dagger}=2^{-1 / 2}\left(b_{\alpha x}^{\dagger} \pm i b_{\alpha y}^{\dagger}\right)$ and $b_{\alpha_{ \pm}}=2^{-1 / 2}\left(b_{\alpha x} \mp i b_{\alpha y}\right)$, to the light intensity $E^{ \pm}(t)$ of the same polarization. The third term denotes decay of these IR phonons, with wave vectors $k \approx 0$, into phonon pairs with opposite wave vectors $\pm k$, which most likely come from the same acoustical branch 13, 14,. These also can not carry angular quasi-momentum $L$, which is passed to the tube by umklapp processes. The resulting tube rotation is predominantly damped by scattering with molecules, as described in $H_{d}$ [15].

In Fig. 2, we show two (doubly degenerate) IR modes in the elementary cell, with 40 atoms, of the $(10,10)$ 


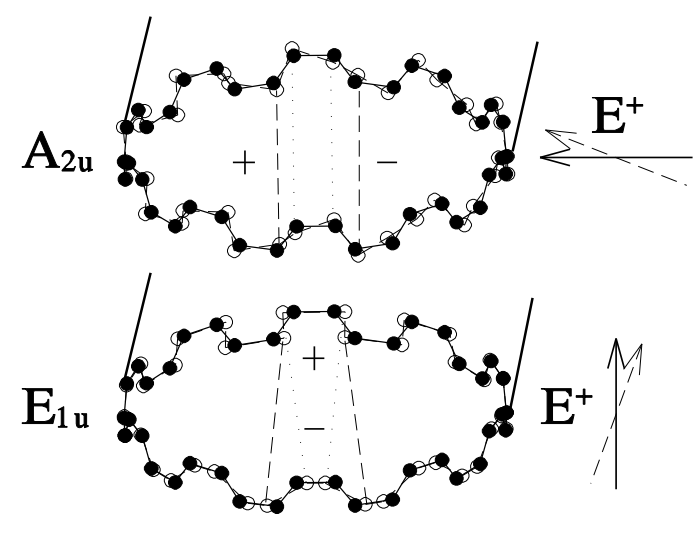

FIG. 2. Nanotube cross-sections with light-induced atomic displacements (open circles) from equilibrium positions (filled circles) in two IR phonon modes, with the $A_{2 u}$ and $E_{1 u}$ symmetries. A circularly polarized light, $\mathbf{E}^{+}$, excites phonon waves, which propagate uni-directionally on the tube circumference in phase with the light polarization.

nanotube. In the $A_{2 u}$ and $E_{1 u}$ modes, the atoms move out-of-plane and in-plane, respectively, orthogonal to the tube axis [16], as shown by open circles. Combination of the two degenerate linearly polarized modes forms a circularly polarized phonon mode, of either symmetry, which can absorb angular quasi-momentum from circularly polarized photons. The atomic displacements break the tube symmetry and induce electric dipoles $(+-)$, which follow in time the polarization of the circulating electric field $\mathbf{E}^{+}$. The effect does not rely on coherent light and can be also realized in tubular rings (see Fig. 1).

As an example, we consider excitation of the $A_{2 u}$ mode. The total number $n_{A_{2 u}}^{+}$of circularly polarized phonons, excited in the vicinity of $k=0$, is given by the Boltzmann equation

$$
\frac{\partial n_{A_{2 u}}^{+}}{\partial t}=\dot{n}_{A_{2 u}}^{+}-\frac{n_{A_{2 u}}^{+}-n_{A_{2 u} ; \text { equil. }}^{+}}{\tau_{A_{2 u}}} .
$$

$\dot{n}_{A_{2 u}}^{+}$is their injection rate and $\tau_{A_{2 u}}=2 \hbar / \gamma_{A_{2 u}} \approx 2 \mathrm{ps}$, is the relaxation time, where $\gamma_{A_{2 u}} \approx 22 \mathrm{~cm}^{-1}$ is the width of the IR phonon lines in nanotubes [10]. We neglect small populations $n_{A_{2 u}}^{-}$of phonons with the opposite polarization, resulting in scattering.

The absorption line of the $A_{2 u}\left(E_{1 u}\right)$ mode was observed near $\omega_{A_{2 u}}=870 \mathrm{~cm}^{-1}\left(1580 \mathrm{~cm}^{-1}\right)$ in both graphite and $\mathrm{C}$ nanotubes. In graphite, the $A_{2 u}$ mode has an oscillator strength [9] $f \approx 0.004$, which we assume to approximately hold in $\mathrm{C}$ nanotubes. Its optical dipole moment is 17] $\mu_{A_{2 u}}=e \sqrt{3 \hbar f / 2 m_{\text {osc }} \omega_{A_{2 u}}} \approx$ $10^{-31} \mathrm{Cm}$, where $m_{\text {osc }}=M_{\text {Carbon }} / 2$ is the oscillator mass. Using the Fermi's Golden rule, and assuming that $n_{A_{2 u} ; \text { equil. }}^{+} \approx 0$, we obtain the injection rate

$$
\dot{n}_{A_{2 u}}^{+} \approx \frac{2 \pi}{\hbar}\left|\mu_{A_{2 u}} E^{+}\right|^{2} \rho\left(\omega_{A_{2 u}}\right)
$$

where $\rho\left(\omega_{A_{2 u}}\right)$ is the density of phonon modes at $k=0$. An armchair $(10,10)$ nanotube of length $l=1 \mu \mathrm{m}$ has $n \approx 1.6 \times 10^{5} \mathrm{C}$ atoms and $N=n / 40=4000$ elementary cells $\left(A_{2 u}\right.$ modes with $\left.k \neq 0\right)$. About $10 \%$ of these modes (around $k=0$ ) fall in the energy window $\gamma_{A_{2 u}}$, thus giving the effective mode density $\rho\left(\omega_{A_{2 u}}\right) \approx 400 / \gamma_{A_{2 u}}$. For a field strength $E^{+}=10 \mathrm{kV} / \mathrm{m}$, we then obtain from Eq. 3 that $\dot{n}_{A_{2 u}}^{+} \approx 2.5 \times 10^{5} \mathrm{~s}^{-1}$. The IR phonons thus absorb the angular quasi-momentum with the rate $\dot{L}_{A_{2 u}}=\hbar \dot{n}_{A_{2 u}}^{+} \approx 2.5 \times 10^{-29} \mathrm{Nm}$.

We can understand the angular quasi-momentum umklapp processes by unrolling the nanotube, and loosely binding many such sheets into a superlattice of lattice constant $a_{s}=2 \pi r$, where $r$ is the tube diameter. Then, the IR phonons modes have the transversal wave vector $K_{0}=2 \pi / a_{s}$, which falls in the middle of the second Brillouin mini-zone of size $Q=K_{0}$. In a twophonon umklapp decay, the momentum conservation is $K_{0}+K_{1}+K_{2}=Q$ (transversal wave vectors of the decayed acoustical phonons are $K_{1,2}=0$ ), where the vector $Q$ interconnects centers of the first and second mini-zone. In the nanotube, we can vector multiply this identity by $\hbar r$, and obtain the (umklapp) angular quasi-momentum conservation $L_{0}+L_{1}+L_{2}=\hbar Q \times r\left(L_{1,2}=0\right)$, where $L_{0}=\hbar K_{0} \times r \equiv \hbar$.

In Eq. 2 these processes are represented by the relaxation time $\tau_{A_{2}, \text { }}$ which could be derived from Eq. 1 following Klemens [13]. Its experimental value, $\tau_{A_{2 u}} \approx 2 \mathrm{ps}$, is in agreement with decay times of the suggested processes realized in other systems [14]. Since $a b$ initio calculations of the phonon matrix elements $c_{\alpha_{ \pm}, \beta, \gamma}$ are lacking, we use this value of $\tau_{A_{2 u}}$ in our modeling. Eq. 2 2 then gives the steady-state angular quasi-momentum in the $A_{2 u}$ phonon bath $L_{A_{2 u}}=\hbar \Delta n_{A_{2 u}}^{+}=\dot{L}_{A_{2 u}} \tau_{A_{2 u}} \approx 5.2 \times 10^{-41} \mathrm{Js}$.

The angular momentum is transferred to the tube body at the rate $\dot{L} \approx \dot{L}_{A_{2 u}}$. Nanotubes in liquids 15] or under atmospheric conditions would rotate slowly, since collisions with the surrounding molecules quickly dissipate the acquired angular momentum. On the other hand, in low-vacuum environment, with realistic collisional rates $\kappa \approx 10^{-12}-10^{-13} \mathrm{~cm}^{3} \mathrm{~s}^{-1}$, damping times of the order $\tau_{\text {damp }} \approx 10 \mathrm{~s}$ are readily achievable. The tube thus keep a steady-state angular momentum $L \approx \dot{L}_{A_{2 u}} \tau_{\text {damp }} \approx 2.5 \times 10^{-28}$ Js.

The nanotube rotation frequency $\omega_{\text {rot }}$ can be found upon calculating its principal moments of inertia 18 20]

$$
A=B=M\left(\frac{r_{e}^{2}+r_{i}^{2}}{4}+\frac{l^{2}}{12}\right), C=M \frac{r_{e}^{2}+r_{i}^{2}}{2} .
$$

Here $M=\rho l, r_{e}, r_{i}$, and $l$ are the nanotube mass ( $\rho$ is the linear density), exterior and interior radii, and length, respectively. For the $(10,10)$ armchair nanotube with $r=\left(r_{e}+r_{i}\right) / 2 \approx 0.68 \mathrm{~nm}$ and $l=1 \mu \mathrm{m}$, we obtain $M \approx$ $1.9 \times 10^{-20} \mathrm{~kg}$ and $A \approx 1.6 \times 10^{-33} \mathrm{~kg} \mathrm{~m}^{2} \approx 1.8 \times 10^{5} C$.

Finally, we find the rotation speed $\omega_{\text {rot }}=L / C \approx 28$ $\mathrm{GHz}$ for this elementary nano-mechanical device. Centrifugal acceleration on its surface is enormous, $a=$ 
$r \omega_{\text {rot }}^{2}=0.5 \times 10^{12} \mathrm{~m} / \mathrm{s}^{2} \approx 10^{11} \mathrm{~g}$. This value surpasses by two orders of magnitude the acceleration obtained with sub-millimeter steel balls [21], and by five orders of magnitude acceleration in the fastest centrifuges [22]. Since for $a=10^{11} \mathrm{~g}$ the force on each $\mathrm{C}$ atom, $F \approx 13 \mu \mathrm{eV} / \AA$ is still negligible with respect to chemical forces $(1 \mathrm{eV} / \AA)$, the tube rotation could be further increased. On this path to "tera-gravity", unique parameters of nanotubes can play a pivotal role.

We can now discuss in more details practical spinning experiments. Isolated SWNT or MWNT have been grown, for example, on an AFM tip [23], which can be later placed inside an optical trap. The nanotube can be severed from the tip using, for instance, a focused electron beam 24. Detached tubes could be also transported to the trap by recently developed nano-tweezers 25]. The optical trap can be formed by two linearly and mutually parallel polarized counter-propagating laser beams [26.

Nonresonant scattering of trap-beam photons from the nanotube with polarizability $\alpha$ produces a force, oriented in the direction of increasing light intensity $I$, that results in the potential $U=-\alpha I / 2 c$ ( $c$ is the speed of light). The longitudinal $\alpha_{z z} \approx 500 \AA^{3} /$ atom and radial $\alpha_{x x} \approx 25 \AA^{3}$ /atom static polarizabilities of semiconducting nanotubes [27] are quite different, and this difference is even larger in metallic tubes. Therefore, the tube in the trap remains oriented along the beam polarization axis, where it experiences the trapping potential

$$
U \approx-U_{0} e^{-r^{2} / \sigma_{r}^{2}-z^{2} / \sigma_{z}^{2}} \approx-\left(U_{0}-S r^{2}\right) e^{-z^{2} / \sigma_{z}^{2}} .
$$

Here $x, y, z\left(r=x^{2}+y^{2}\right)$ are the tube center-of-mass coordinates, and $S=S_{0} l=U_{0} / \sigma_{r}^{2}$ is the trap rigidity. To prevent thermal escape of the tube from the trap, we consider a trap depth $U_{0}=n \alpha_{z z} I / 2 c \approx 10 \mathrm{eV}$, and obtain $I \approx 1.2 \mathrm{GW} / \mathrm{cm}^{2}$. The trap laser frequency must be below the band gap, $E_{g} \approx 1 \mathrm{eV}$, and away from the frequencies of the tube internal modes, see below. Assuming that $\sigma_{r} \approx 1 \mu \mathrm{m}$, we find $S_{0}=U_{0} / l \sigma_{r}^{2} \approx 1.6 \mathrm{~J} / \mathrm{m}^{3}$.

Small amounts of defects and adsorbants on the tube walls do not prevent its spinning, but can shift its rotation frequency. In accordance with the De Laval principle of self-balancing [20], such a partially-coated nanotube floating in the trap would rotate around an eccentric axis. Rapid rotation of the nanotube can be also limited by its mechanical vibrations in the trap, as discussed below. To avoid its large oscillations, the critical frequencies should be quickly passed during the acceleration [19,20].

The cylindrical whirl mode [20] reflects the rigid-body vibrations of the tube orthogonal to the trap axis. The forward (backward) cylindrical frequencies are $\omega_{c y l}=$ $\pm \sqrt{S_{0} / \rho} \approx \pm 9.2 \mathrm{MHz}$. In the conical whirl mode, the tube ends move in opposite directions with respect to the tube/trap axis. For a tube distorted through the angle $\theta$, the torsional moment is $M_{F} \approx-S_{0} l^{3} \theta / 6$, resulting in the Euler's equation, 119] $A \omega_{\text {con }}^{2}=C \omega_{\text {rot }} \omega_{\text {con }}+S_{0} l^{3} / 6$. Using $A \approx \rho l^{3} / 12$, valid for $l \gg r$, we obtain

$$
\omega_{c o n}=\frac{C \omega_{r o t}}{2 A} \pm \sqrt{\left(\frac{C \omega_{r o t}}{2 A}\right)^{2}+\frac{2 S_{0}}{\rho}} .
$$

We can see that the modal frequencies depend on $\omega_{\text {rot }}$ due to gyroscopic effects [20]. Since the ratio $C / A \approx l^{-2}$ is small, the effects are suppressed by the potential $U$, so that $\omega_{\text {con }} \approx \sqrt{2} \omega_{c y l}$. From Eq. 6, we find that they begin to play a role for tube lengths $l<\left(r_{e}+r_{i}\right) \sqrt{3 \omega_{\text {rot }} / \omega_{c y l}} \approx$ $130 \mathrm{~nm}$. If the trap is suddenly switched off, a micronlong nanotube rotating with frequency $\omega_{\text {rot }}=28 \mathrm{GHz}$, and initially disturbed on its side, would precess with the frequency $\omega_{\text {prec }}=C \omega_{\text {rot }} / A \approx 175 \mathrm{kHz}$.

In long nanotubes, one needs to consider also flexural vibrations [18 20]. The critical flexural frequencies $\omega_{f}$ can be evaluated from the equations for lateral deflections $x(z), y(z)$ at different points $z$ along the trap axis, if the rigid body approximation is abandoned. The equation for the $x$ deflection is

$$
\begin{aligned}
Y I \frac{\partial^{4} x}{\partial z^{4}} & =-\rho \frac{\partial^{2} x}{\partial t^{2}}-S_{0} x \\
& +a \frac{\partial^{2}}{\partial t^{2}}\left(\frac{\partial^{2} x}{\partial z^{2}}\right)+c \omega_{\text {rot }} \frac{\partial}{\partial t}\left(\frac{\partial^{2} y}{\partial z^{2}}\right) .
\end{aligned}
$$

Here $Y$ is the Young modulus, $I=\pi\left(r_{e}^{4}-r_{i}^{4}\right) / 4$ is the second moment of nanotube cross-section and the factors $c=2 a=\rho\left(r_{e}^{2}+r_{i}^{2}\right) / 2$ are the densities of the moments of inertia 19, which correspond to the bulk expressions in Eq. 4 in the limit $l \rightarrow 0$. The equation for the $y$ deflection results from Eq. 7 by exchanging $x \leftrightarrow y$ and a negative sign in the last term.

The flexural frequencies correspond to the solutions $x=x_{0} \cos \left(\omega_{f} t\right), y=y_{0} \sin \left(\omega_{f} t\right)$ in Eq. 7. This substitution gives an ordinary differential equation, identical for both the $x$ and $y$ deflections. For simplicity, we apply the clamped-end approximation, with the boundary conditions $x_{0}\left(z= \pm \frac{l}{2}\right)=d^{2} x_{0}\left(z= \pm \frac{l}{2}\right) / d z^{2}=0$. The solutions are $x_{0}(z)=A_{0} \cos (\xi z)$ or $x_{0}(z)=A_{0} \sin (\xi z)$, where $\xi^{2}=\left(\alpha+\sqrt{\alpha^{2}-4 \beta Y I}\right) / 2 Y I, \alpha=a \omega_{f}^{2}-$ $c \omega_{\text {rot }} \omega_{f}$, and $\beta=S_{0}-\rho \omega_{f}^{2}$. Therefore, $\xi=n \pi / l$, with $n=1,2,3, \ldots$ indexing the eigenmodes, which leads to the critical flexural frequencies $\left(\omega_{f n}=\omega_{\text {rot }}\right)$

$$
\omega_{f n}=\sqrt{\frac{S_{0} / \rho+(n \pi / l)^{4} Y I / \rho}{1-(n \pi r / l)^{2} / 2}} .
$$

We use the values $Y \approx 5.5 \mathrm{TPa}$ and $h=r_{e}-r_{i}=0.066$ $\mathrm{nm}$, found in molecular dynamics simulations [28].

In Fig. 3, we show the dependence of the lowest critical frequencies $\omega_{f n}$ on the tube length $l$, calculated from Eq. 8 using the numerical values for $Y, h, \rho, S_{0}$ and $r$. For long tubes, the frequencies $\omega_{f n}$ coincide with $\omega_{c y l}$, while for shorter tubes $(l<1.3 \mu \mathrm{m})$, the bending term surpasses the trap term, and $\omega_{f n}=(n \pi / l)^{2} \sqrt{Y I / \rho}$. In the continuum description, gyroscopic effects become only important for high eigenmodes $n \approx l / r$. In the inset of Fig. 3, we also show the dependence of $\omega_{f n}$ on $n$ for 


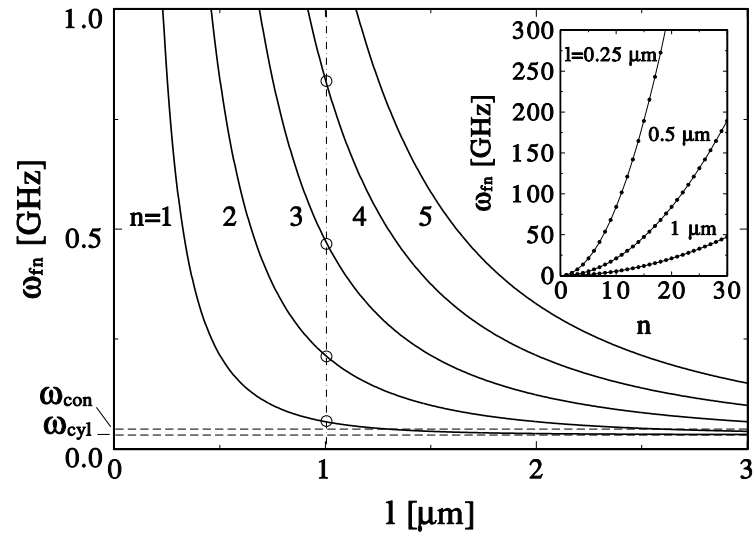

FIG. 3. Dependence of the critical flexural frequencies $\omega_{f n}$ on the nanotube length $l$. The two thin horizontal dashed lines correspond to $\omega_{c y l}$ and $\omega_{c o n}$. In the inset, we show $\omega_{f n}$ as a function of the bending modal number $n$ for nanotubes of different length.

tubes of different lengths. The huge Young modulus $Y$ makes the density of critical frequencies $\omega_{f n}$ relatively low, especially for short nanotubes. This allows for a rapid traversal to the "supercritical state", which is realized above the flexural or other vibrational frequencies.

Rotating nanotubes could form parts of nano-motors, centrifuges or stabilizers. Centrifugal studies could be performed inside microtubes with large diameters $d \approx$ $10 \mu \mathrm{m}$ [29] or in assemblies made from nanotube rings, forming strong but flexible skeletal coats. One could also think about possible applications of rotating tubes in liquids. Slowly rotating coiled nanotubes [30] could, for example, propel microscopic systems, which would chemically power the rotation of these tubes that attached to their surfaces in bearings [31], as in bio-motors. We believe that unique properties of nanotubes made from carbon and other materials could foster applications with rotating micro-elements.

We would like to thank R. Saito and G. Dresselhaus for data on IR phonon modes and several useful discussions. PK would like to acknowledge support from EU COCOMO. This work was also supported by the US National Science Foundation through a grant to the Institute for Theoretical Atomic and Molecular Physics at the Harvard-Smithsonian Center for Astrophysics.

[1] S. Iijima, Nature 354, 56 (1991).

[2] M. S. Dresselhaus, G. Dresselhaus, and P. C. Eklund, Science of Fullerenes and Carbon Nanotubes (Academic Press Inc., San Diego, 1996).
[3] M. M. Treacy, T.W. Ebbesen and J. M. Gibson, Nature 381, 678 (1996); R. Gao et al., Phys. Rev. Lett. 85, 622 (2000).

[4] O. Lourie, D. M. Cox and H. D. Wagner, Phys. Rev. Lett. 81, 1638 (1998).

[5] D. Walz and S. R. Caplan, Biophys. J. 78, 626 (2000). F. A. Samatey et al., Nature 410, 331 (2001).

[6] J. Karczmarek, J. Wright, P. Corkum and M. Ivanov, Phys. Rev. Lett. 82, 3420 (1999); D. M. Villeneuve et al., Phys. Rev. Lett. 85, 542 (2000).

[7] E. Santamato, B. Daino, M. Romagnoli, M. Settembre and Y. R. Shen, Phys. Rev. Lett. 57, 2423 (1986).

[8] E. Higurashi et al., Appl. Phys. Lett. 64, 2209 (1994); L. Paterson et al., Science 292, 912 (2001).

[9] R. J. Nemanich, G. Lucovsky and S. A. Solin, Sol. St. Commun. 23, 117 (1977).

[10] J. Kastner et al., Chem. Phys. Lett. 221, 53 (1994); U. Kuhlmann, H. Jantoljak, N. Pfander, P. Bernier, C. Journet and C. Thomsen, Chem. Phys. Lett. 294, 237 (1998);

[11] J. Liu et al., Nature 385, 780 (1997).

[12] A. A. Kiselev, Opt. Spectrosc. 53, 469 (1982).

[13] P. G. Klemens, in Solid State Physics, ed. by F. Seitz and D. Turnbull, (Academic Press Inc., NY, 1958), Vol. 7; P. G. Klemens, Phys. Rev. 148, 845 (1966).

[14] S. Usher and G. P. Srivastava, Phys. Rev. B 50, 14179 (1994).

[15] P. Král and M. Shapiro, Phys. Rev. Lett. 86, 131 (2001).

[16] R. Saito (private communication).

[17] R. W. Boyd, Nonlinear Optics, (Academic Press, 1992).

[18] L. D. Landau and E. M. Lifshitz, Elasticity Theory (Pergamon, Oxford, 1986).

[19] R. N. Arnold and L. Maunder, Gyrodynamics and its Enginering Applications, (Academic, NY/London 1961).

[20] S. Whitley, Rev. Mod. Phys. 56, 41 (1984); ibid 56, 67 (1984).

[21] J. W. Beams, Sci. Am. 204, 134 (1961).

[22] T. Mashimo, S. Okazaki and S. Shihabazaki, Rev. Sci. Instrum. 67, 3170 (1996).

[23] Ch. L. Cheung, J. H. Hafner, T. W. Odom, K. Kim and C. M. Lieber, Appl. Phys. Lett. 76, 3136 (2000).

[24] S. Trasobares et al., Eur. Phys. J. B 22, 117 (2001).

[25] P. Kim and C. M. Lieber, Science 286, 2148 (1999).

[26] A. Ashkin, Phys. Rev. Lett. 24, 156 (1970); M. J. Renn, R. Pastel and H. J. Lewandowski, Phys. Rev. Lett. 82, 1574 (1999).

[27] L. X. Benedict, S. G. Louie, and M. L. Cohen, Phys. Rev. B 52, 8541 (1995).

[28] B. I. Yakobson, C. J. Brabec and J. Bernholc, Phys. Rev. Lett. 76, 2511 (1996).

[29] M. Remškar et al., Appl. Phys. Lett. 69, 351 (1996).

[30] X. B. Zhang et al., Europhys. Lett. 27, 141 (1994); A. Volodin et al., Phys. Rev. Lett. 84, 3342 (2000).

[31] A. N. Kolmogorov and V. H. Crespi, Phys. Rev. Lett. 85, 4727 (2000); J. Cumings and A. Zettl, Science 289, 602 (2000). 